\title{
Efficient laser textured nanocrystalline silicon-polymer bilayer solar cells
}

\author{
A. A. D. T. Adikaari, ${ }^{\text {a) }}$ D. M. N. M. Dissanayake, R. A. Hatton, and S. R. P. Silva \\ Nano Electronics Centre, Advanced Technology Institute, University of Surrey, Guildford, Surrey GU2 7XH, \\ United Kingdom
}

(Received 30 March 2007; accepted 19 April 2007; published online 18 May 2007)

\begin{abstract}
Excimer laser textured thin film silicon and poly(2-methoxy-5-(2'-ethylhexyloxy)-1,4-phenylenevinylene bilayer solar cells are fabricated and characterized with air mass 1.5 simulated solar irradiation. The polymer layer increases the light harvesting capability of the cell and increases the shunt resistance while increasing open circuit voltage. The highest efficiency of $0.87 \%$ for the thin film silicon/polymer bilayer device is observed due to enhanced charge collection resulting from the inclusion of the polymer layer. Generation of charge carriers is proposed to be occurring mainly in the silicon layer and charge transport is explained using an energy band diagram. () 2007 American Institute of Physics. [DOI: 10.1063/1.2739365]
\end{abstract}

Organic photovoltaics have been extensively investigated over the past few years due to their potential low cost nature and envisaged simplicity in fabrication. ${ }^{1}$ Interest in organic/inorganic heterojunctions has grown simultaneously with most of the work concentrated on titania based photovoltaics. $^{2}$ A small number of reports pertaining to hydrogenated amorphous silicon $(a-\mathrm{Si}: \mathrm{H})$ /polymer heterojunction solar cells have been reported. ${ }^{3,4}$ However, active participation of the polymer in photocurrent generation has been confirmed only recently. ${ }^{4}$ The efficiency of these hybrid devices has been low, and for $a-\mathrm{Si}: \mathrm{H} /$ polymer cell, the best reported efficiency is $0.01 \%$. $^{4}$

Excimer lasers have been utilized for the crystallization of $a$-Si: $\mathrm{H}$ for electronic applications ${ }^{5,6}$ with well established crystallization physics. ${ }^{7-9}$ Excimer lasers typically operate in the ultraviolet and hence photons are absorbed by the silicon thin films within a few nanometers of the surface. Melting and solidifying occur on a nanosecond time scale, often without affecting the underlying substrate. The properties of crystallized silicon depend on a number of factors, with laser pulse shape being one of the most significant. The surface of the laser crystallized silicon film can be extremely rough, ${ }^{6}$ and by changing the beam shape, the film can be nanostructured simultaneously during crystallization to create a large area textured nanocrystalline silicon (nc-Si:H) for use in nc$\mathrm{Si}: \mathrm{H}$ /polymer heterojunction photovoltaic devices. We report laser crystallized and textured nc-Si:H/poly(2-methoxy5-(2'-ethyl-hexyloxy)-1,4-phenylenevinylene, (MEH-PPV) bilayer solar cell, which shows significantly higher efficiency compared to previously reported $a-\mathrm{Si}: \mathrm{H} / \mathrm{MEH}-\mathrm{PPV}$ solar cells.

Chemical vapor deposited heavily doped $p$-type hydrogenated amorphous silicon carbide $(\sim 12 \mathrm{~nm}) /$ undoped $a-\mathrm{Si}: \mathrm{H}(400 \mathrm{~nm})$ structures deposited on $500 \mathrm{~nm}$ thick indium tin oxide (ITO) coated glass substrates were obtained from a commercial producer. A KrF Lambda Physik excimer laser (LPX 210i), operating at $248 \mathrm{~nm}$ with a $25 \mathrm{~ns}$ full width at half maximum pulse duration, was used for laser nanostructuring. ${ }^{6}$ The $a$-Si: $\mathrm{H}$ films on glass were nanostructured in air by scanning along an asymmetric peak 4

${ }^{a}$ Author to whom correspondence should be addressed; electronic mail: d.adikaari@surrey.ac.uk $\times 8 \mathrm{~mm}^{2}$ laser pulse. The scanning was performed along the long axis, keeping the steeper edge of the asymmetric pulse as the leading edge. The scanning speed for the experiment was $2 \mathrm{~mm} \mathrm{~s}^{-1}$ with pulse repetition rate maintained at $50 \mathrm{~Hz}$. An energy density of $160 \mathrm{~mJ} \mathrm{~cm} \mathrm{~cm}^{-2}$ pulse was used for nanostructuring. The laser energy was measured using a Molectron EPM 1000 analyzer and a J25LP-MUV pyroelectric detector.

The laser nanostructured device subassembly was then dipped into a buffered hydrofluoric acid for $20 \mathrm{~s}$ to remove the oxide layer. A $10 \mathrm{mg} \mathrm{cm}^{-3} \mathrm{MEH}-\mathrm{PPV}$ in chloroform was spun cast onto the subassembly at $1500 \mathrm{rpm}$ for $60 \mathrm{~s}$ immediately after the acid treatment, before evaporating a $50 \mathrm{~nm}$ thick Al back electrode. A similarly textured, acid treated sample was investigated along with a polymer spun sample, using atomic force microscopy (AFM) for surface characterization. Figure 1(a) shows a three dimensional representation of a $5 \times 5 \mu \mathrm{m}^{2}$ area of oxide removed textured surface. The rms roughness of this surface was calculated to be $257 \mathrm{~nm}$, whereas the rms roughness of polymer spun surface was $174 \mathrm{~nm}$. Figure 1(b) shows the three dimensional representation of the polymer spun surface. Immediately after Al
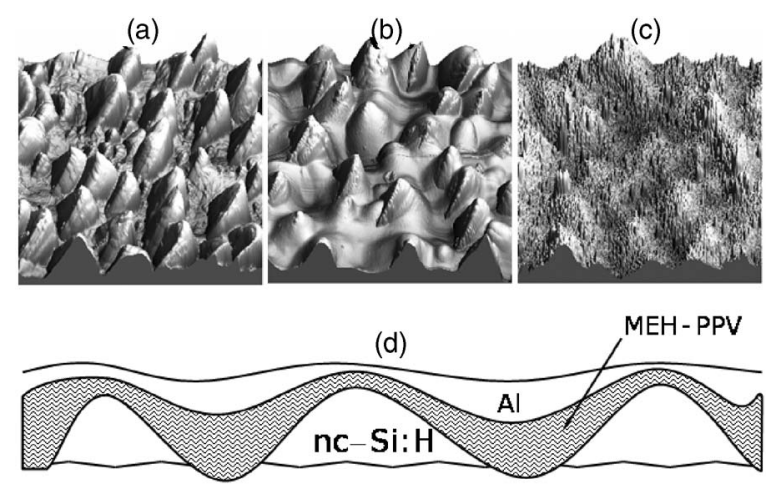

Пто

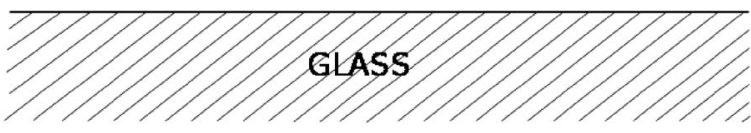

FIG. 1. Three dimensional representation of a $5 \times 5 \mu \mathrm{m}^{2}$ area of (a) laser textured silicon surface, after oxide removal, (b) polymer spun surface, and (c) Al electrode surface, obtained from atomic force microscopy. (d) Schematic diagram of the nc-Si:H/MEH-PPV bilayer solar cell. 


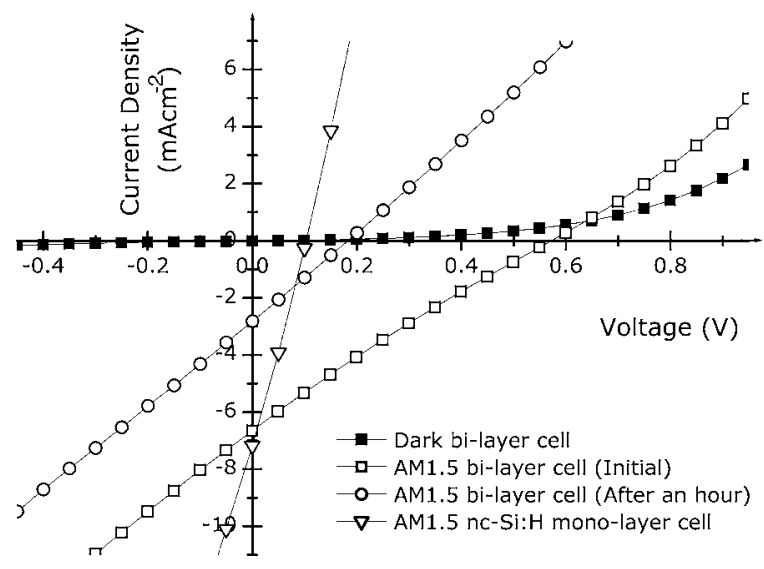

FIG. 2. Current density-voltage characteristics of the nc-Si:H/MEH-PPV bilayer cell and nc-Si:H monolayer cell. Photovoltaic characteristics under AM1.5 simulated solar irradiation for bilayer device immediately after fabrication shown by hollow squares and after an hours' exposure to atmosphere in the dark, shown by circles. Current density-voltage characteristics of the bilayer device in the dark is shown by black squares. Photovoltaic characteristics under AM1.5 simulated solar irradiation for a nc-Si:H monolayer cell is shown by triangles.

evaporation creating devices with a $5-8 \mathrm{~mm}^{2}$ area, they were characterized with air mass $1.5\left(1000 \mathrm{~W} \mathrm{~m}^{-2}\right)$ simulated solar irradiation. Photovoltaic current-voltage characteristics were measured using an Oriel 81160 Class B (Ref. 10) solar simulator with a nonuniformity of irradiance at $\leqslant 5 \%$. ${ }^{11}$ A Keithley 2400 source meter was used for the current-voltage measurements. The photovoltaic currentvoltage characteristics were measured again after an hour, before probing the $\mathrm{Al}$ surface using AFM. Figure 1(c) shows the three dimensional representation of the Al surface, which resulted in a rms roughness of $21 \mathrm{~nm}$.

From AFM, the maximum peak-to-peak height of the surface protrusions of the laser nanostructured surface was calculated to be greater than a micron, reduced to just below a micron for the polymer spun surface. It is further reduced to $\sim 100 \mathrm{~nm}$ for the Al surface. Since the thickness of the initial $a$-Si:H film is $400 \mathrm{~nm}$, these heights suggest that the texturing process exposes the underlying ITO layer at random locations. It is assumed that the bulk of the area is covered with at least a very thin layer of nc-Si:H. A schematic diagram of the bilayer solar cell based on these observations is shown in Fig. 1(d). The thickness of the polymer layer of the device is nonuniform, as seen in Fig. 1(d), while the effective captive area of the solar irradiation is increased. The nc-Si:H layer is expected to be similar to that of a partially melted film with a stratified structure. ${ }^{7}$

Figure 2 shows the current density-voltage characteristics of the solar cell measured in the dark (black squares) and under simulated solar irradiation (hollow squares), immediately after fabrication. The cell has an open circuit voltage $\left(V_{\mathrm{oc}}\right)$ of $0.56 \mathrm{~V}$ and a short circuit current density $\left(J_{\mathrm{sc}}\right)$ of $6.65 \mathrm{~mA} \mathrm{~cm}^{-2}$. The fill factor of the device $(0.23)$ is low, owing to the high series and low shunt resistances, which is evident from the gradients at forward bias and at $J_{\mathrm{sc}}$, respectively. The resultant power conversion efficiency of the device is $0.87 \%$, which is two orders of magnitude greater than the best $a$-Si:H/MEH-PPV cell reported to date. ${ }^{4}$ The MEHPPV layer of the device shows an electron acceptorlike behavior in this case, which is contrary to previous observations. ${ }^{4}$
In order to check the stability of the device, the device current-voltage characteristics were measured under simulated irradiation, after an hour's exposure in the dark to atmospheric conditions. The performance degraded drastically, as shown in Fig. 2 (circles), with $V_{\mathrm{oc}}$ and $J_{\mathrm{sc}}$ reduced to $0.19 \mathrm{~V}$ and $2.77 \mathrm{~mA} \mathrm{~cm}^{-2}$, respectively, resulting in a power conversion efficiency of $0.13 \%$. MEH-PPV based organic solar cells are highly susceptible to atmospheric exposure, degrading the photocurrent density with commensurate decrease in efficiency. ${ }^{12}$ It has been reported that the current density of MEH-PPV homopolymer devices is reduced by $80 \%$ during the first hour of atmospheric exposure. ${ }^{12}$ With the devices analyzed here, $J_{\mathrm{sc}}$ is reduced by $60 \%$ after an hour's exposure to atmosphere, comparing better and suggesting that the polymer layer is active during the initial operation of the device.

A laser crystallized nc-Si:H reference device without the MEH-PPV layer is impossible to be fabricated using exactly the same crystallization/nanostructuring conditions due to pinhole creation. However, a laser crystallized nc-Si:H cell was fabricated at the same laser energy density for comparison, avoiding pinholes by keeping the surface roughness to a minimum. This condition was achieved using the same $a$-Si:H based subassembly, annealed in vacuum $(0.03 \mathrm{~Pa})$, scanning with the asymmetric pulse utilizing the gradual end as the leading edge. All other parameters were maintained identical to the bilayer device fabrication process. The nc$\mathrm{Si}: \mathrm{H}$ monolayer cell contains more silicon compared to the laser textured nc-Si:H film, due to less ablation during crystallization, resulting in a continuous film with a rms roughness of $45 \mathrm{~nm}$. The cell completed with $\mathrm{Al}$ back contact yielded a power conversion efficiency of $0.20 \%$, with $V_{\mathrm{oc}}$ $0.10 \mathrm{~V}$ and $J_{\mathrm{sc}} 7.20 \mathrm{~mA} \mathrm{~cm}^{-2}$. The current density-voltage characteristics of the nc-Si:H monolayer cell under simulated irradiation are shown in Fig. 2 (triangles).

The shunt resistance $\left(R_{\mathrm{sh}}\right)$ of the nc-Si:H monolayer cell was low $(298 \Omega)$, believed to be due to grain boundaries increasing the leakage at thin areas of the active layer from laser annealing. However, the $R_{\mathrm{sh}}$ of the nc-Si:H/MEH-PPV cell shows a threefold increase with the inclusion of the MEH-PPV layer at $970 \Omega$. The bilayer device $R_{\text {sh }}$ also shows reasonable stability for atmospheric exposure, showing $870 \Omega$ after degradation. The $R_{\text {sh }}$ improvement from the inclusion of the MEH-PPV layer is identified to be one of the reasons for the higher efficiency of bilayer device.

The photon conversion efficiencies of the hybrid cell and nc-Si:H monolayer cell were measured just after the initial photovoltaic characterization. A Bentham IL1 $100 \mathrm{~W}$ halogen light source and a TMc 300 monochromator were used along with a Keithley 487 picoammeter for measurements. The incident optical power onto the sample was measured with a calibrated Newport 1830C optical power meter. Figure 3 shows the photon conversion efficiencies of the bilayer device (squares) and nc-Si:H cell (hollow circles) along with the normalized optical absorption of MEH-PPV (black circles). The nc-Si:H cell shows lower photon conversion efficiencies, mainly believed to be due to thermal damage to the $p$-type/intrinsic silicon interface. ${ }^{13}$ From Fig. 3 , it is clear that the photoexcited carrier collection is improved drastically by the inclusion of MEH-PPV layer. This observation suggests that the MEH-PPV layer not only improves the $R_{\text {sh }}$ of the device, but also enhances carrier collection compared to the nc-Si:H monolayer cell. 


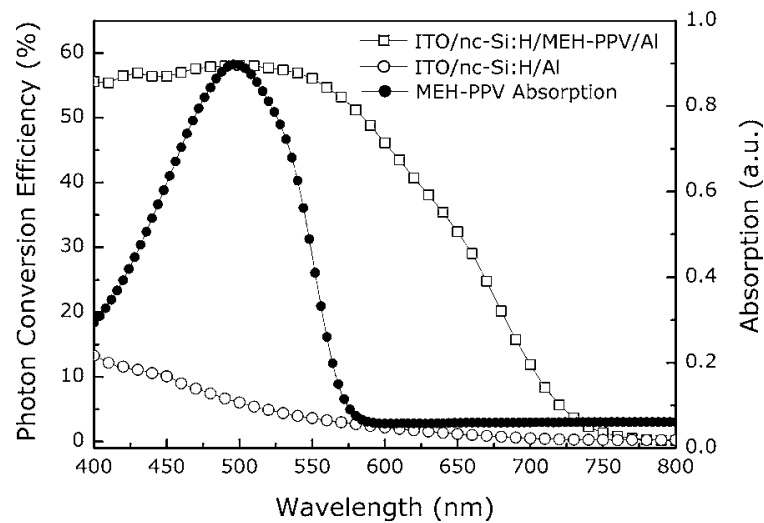

FIG. 3. Photon conversion efficiencies of the nc-Si:H/MEH-PPV bilayer cell (squares), nc-Si:H monolayer cell (hollow circles), and the absorption of MEH-PPV (black circles).

A band diagram for the nc-Si:H/MEH-PPV device was drawn assuming that at the ITO end, nc-Si:H is slightly $p$ type after nanostructuring. The vacuum level of the polymer and $\mathrm{Al}$ layer were assumed to be the same for the diagram which is shown in Fig. 4. The barrier introduced at the nc$\mathrm{Si}: \mathrm{H} / \mathrm{MEH}-\mathrm{PPV}$ interface would be expected to retard electron extraction promoting recombination and degrading cell performance. Hence, for the observed device operation, either the barrier should be lowered or electrons should be able to tunnel through to the $\mathrm{Al}$ contact. Therefore, it is plausible that a vacuum level shift occurs at the MEH-PPV/Al interface, ${ }^{14,15}$ leading to the possibility of a critical thickness of MEH-PPV, where electrons can tunnel through to the $\mathrm{Al}$

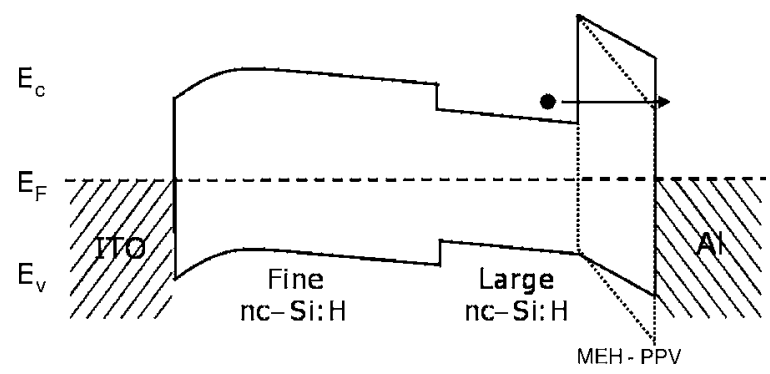

FIG. 4. Band diagram for the laser nanostructured nc-Si:H/MEH-PPV heterojunction cell. Solid lines show a diagram with no vacuum level shifts at $\mathrm{Al}$ and MEH-PPV layers. The dotted section shows the vacuum level shift at MEH-PPV-Al interface, favoring charge transfer across the polymer. contact. The dotted line section in Fig. 4 illustrates this possibility. Further, it has been reported that hole conducting organic layers and low work function metals show Schottkybarrier-like behavior, ${ }^{16}$ which would enhance the charge transport process proposed for the nc-Si:H/MEH-PPV bilayer device.

In conclusion, $0.87 \%$ efficient nc-Si:H/MEH-PPV heterojunction solar cells were fabricated by simultaneous excimer laser crystallization and by texturing an intrinsic $a-\mathrm{Si}: \mathrm{H}$ film. The polymer shows an electron-acceptor-like behavior in organic photovoltaic terminology, instead of a donor, which has been the conventional role of MEH-PPV. The polymer layer increases the light harvesting capability of the cell, and increases the shunt resistance while increasing open circuit voltage. The origin of the photovoltage remains inconclusive and is speculated to be governed by the polymer at a critical thickness in the structure.

The authors thank Nilushan Mudugamuwa for the help provided during device fabrication. This work is supported by the Portfolio Partnership Award from EPSRC.

${ }^{1}$ S. E. Shaheen, D. S. Ginley, and G. E. Jabbour, MRS Bull. 30, 10 (2005).

${ }^{2}$ A. J. Breeze, Z. Schlesinger, S. A. Carter, and P. J. Brock, Phys. Rev. B 64, 125205 (2001).

${ }^{3}$ E. L. Williams, G. E. Jabbour, Q. Wang, S. E. Shaheen, D. S. Ginley, and E. A. Schiff, Appl. Phys. Lett. 87, 223504 (2005).

${ }^{4}$ V. Gowrishankar, S. R. Scully, M. D. McGehee, Q. Wang, and H. M. Branz, Appl. Phys. Lett. 89, 252102 (2006).

${ }^{5}$ S. D. Brotherton, Semicond. Sci. Technol. 10, 721 (1995).

${ }^{6}$ A. A. D. T. Adikaari and S. R. P. Silva, J. Appl. Phys. 97, 114305 (2005).

${ }^{7}$ J. S. Im, H. J. Kim, and M. O. Thompson, Appl. Phys. Lett. 63, 1969 (1993).

${ }^{8}$ N. H. Nickel and K. Brendel, Appl. Phys. Lett. 82, 3029 (2003).

${ }^{9}$ A. A. D. T. Adikaari, J. D. Carey, V. Stolojan, J. L. Keddie, and S. R. P. Silva, Nanotechnology 17, 5412 (2006).

${ }^{10}$ International Electrotechnical Commission Code No. IEC 60904-9, Geneva, Switzerland, 1995.

${ }^{11}$ International Electrotechnical Commission Code No. IEC 904-1, Geneva, Switzerland, 1987.

${ }^{12}$ F. C. Krebs, J. E. Carle, N. Cruys-Bagger, M. Andersen, M. R. Lilliedal, M. A. Hammond, and S. Hvidt, Sol. Energy Mater. Sol. Cells 86, 499 (2005).

${ }^{13}$ L. Jiang, Q. Wang, E. A. Schiff, S. Guha, J. Yang, and X. Deng, Appl. Phys. Lett. 69, 3063 (1996).

${ }^{14}$ H. Ishii, K. Sugiyama, E. Ito, and K. Seki, Adv. Mater. (Weinheim, Ger.) 11, 605 (1999).

${ }^{15}$ Y. Chen, I. Shih, and X. Xiao, J. Appl. Phys. 96, 454 (2004).

${ }^{16}$ H. Hoppe and N. S. Sariciftci, J. Mater. Res. 19, 1924 (2004). 